\title{
Hybrid-Type Fiber Bragg Gratings and Their Applications
}

\author{
Xuewen Shu $^{{ }^{*} \mathrm{a}}$, Kate Sugden ${ }^{\mathrm{a}}$, Donghui Zhao ${ }^{\mathrm{b}}$, Filip Floreani ${ }^{\mathrm{b}}$, Lin Zhang ${ }^{\mathrm{b}}$, Ian Bennion ${ }^{\mathrm{b}}$ \\ a. Indigo Photonics Ltd, Faraday Wharf, Holt Street, Birmingham, B7 4BB, United Kingdom \\ b. Photonics Research Group, Aston University, Birmingham, B4 7ET, United Kingdom
}

\begin{abstract}
Fiber Bragg gratings (FBGs) have attracted a lot of attention in recent years due to their wide applications in optical telecommunications and smart sensing. They have been used as DWDM filters, dispersion compensators, gain flattening filters, optical switch and connection devices, and temperature/strain sensors. FBGs have been found to exhibit four different type structures according to their different growth mechanisms. Each type of FBG exhibits unique thermal and strain properties. Generally, the Type I gratings in hydrogenated and hydrogen-free fibers are used most for applications. However, some novel devices may be achieved by combinational structure of different types of gratings in the future. In this paper, we propose a novel concept of fabrication and application of FBGs with hybrid grating types. We have observed a complex growth behavior of a hybrid-type grating in the UV exposure to a $\mathrm{B} / \mathrm{Ge}$ codoped fiber through a phase mask. A new model has been developed to simulate the complex growth behavior of the hybrid-type gratings, giving results in excellent agreement with experiment.
\end{abstract}

\section{INTRUDUCTION}

Fiber Bragg gratings (FBGs) have attracted a lot of attention in recent years due to their wide applications in optical telecommunications and smart sensing. They have been used as DWDM filters, dispersion compensators, gain flattening filters, optical switch and connection devices, and temperature/strain sensors [1,2]. FBGs have been found to exhibit four different type structures (Type 1, IA, II and IIA) according to their different growth mechanisms $[3,6]$. Each type of FBG exhibits unique thermal and strain properties. Generally, the Type I gratings in hydrogenated and hydrogen-free fibers are used most for applications. However, some novel devices may be achieved by combinational structure of different types of gratings in the future. In this paper, we propose a novel concept of fabrication and application of FBGs with hybrid grating types. We have observed a complex growth behavior of a hybrid-type grating in the UV exposure to a $\mathrm{B} / \mathrm{Ge}$ codoped fiber through a phase mask. A new model has been developed to simulate the complex growth behavior of the hybrid-type gratings.

\section{EXPERIMENT}

In the experiment, a frequency doubled Argon ion laser with Gaussion-profile irradiation of $244 \mathrm{~nm}$ wavelength and a beam

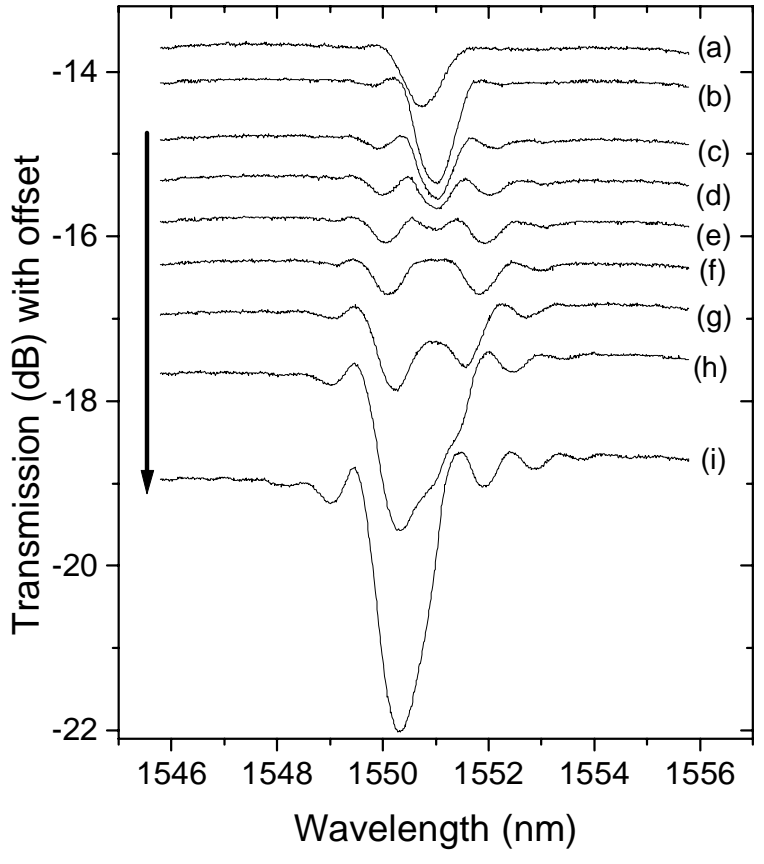

Fig.1 Spectral evolution of a hybrid-type grating under UV exposure.

\footnotetext{
*X.Shu@aston.ac.uk; phone 44121 3593621 5253; fax: 44121 3590156; The work was done with Photonics Research Group, Aston University, Birmingham, B47ET, United Kingdom. Xuewen Shu is now with Indigo Photonics Ltd. Faraday Wharf, Holt Street, Birmingham, B7 4BB, United Kingdom.
} 
size about $0.4 \mathrm{~mm}$ was used. FBGs were inscribed in hydrogen-free B/Ge codoped fiber. In the inscription process, we exposed a beam-size section of the fiber through a phase mask. The laser output power was kept at $100 \mathrm{~mW}$. A broadband light source and an optical spectrum analyzer with a resolution of $0.1 \mathrm{~nm}$ were used to record spectra during UV exposure. Fig.1 show the spectral profiles of the grating evolving from Type I to Type IIA during the UV exposure. Clearly, the grating grew, first, as a normal Type I grating: its strength increases with increasing exposure time and the central wavelength shifts to the longer wavelength side, as shown by the curves (a) and (b) in Fig.1. However, with further increasing UV exposure, the resonance split into three peaks and the center peak grew weaker and eventually disappeared whereas the two outside peaks

became larger, as indicated by the curves (c)-(f). The strengths and bandwidths of the two remaining peaks grew stronger further (curve $(\mathrm{g})$ ) and finally merged into one peak at the short wavelength side (curves $\mathrm{h}$ and I), indicating an almost complete evolution to a Type IIA grating.

\section{SIMULATION AND DISCUSSION}

The experimental observed grating type evolution may be simulated by considering the distribution of the $\mathrm{UV}$-induced refractive index change in a $\mathrm{B} / \mathrm{Ge}$ fiber and the UV beam profile. Although previous publications have concluded that the growth of the Type I grating is associated with a UV-induced positive index change and the Type IIA grating is with a negative index change, the evolving process involving hybrid-type grating has not been discussed. We have developed a new model which combines the effects from the beam profile and the UV induced index distribution. We have found that this model can be used to explain the complete grating evolution process from Type I to Type IIA .

As mentioned in the Experiment section that the UV beam used in the experiment had a Gaussion beam profile. The UV beam with such a profile will lead to the centre part of the grating being exposed more to the laser irradiation than the side parts. For a hydrogen-free $\mathrm{B} / \mathrm{Ge}$ codoped fiber, the grating will change from the type I to type IIA with increasing UV exposure $[3,4]$. Therefore, the centre part first grows into type IIA grating, which means it reaches negative index change, while the side parts remain as type I grating

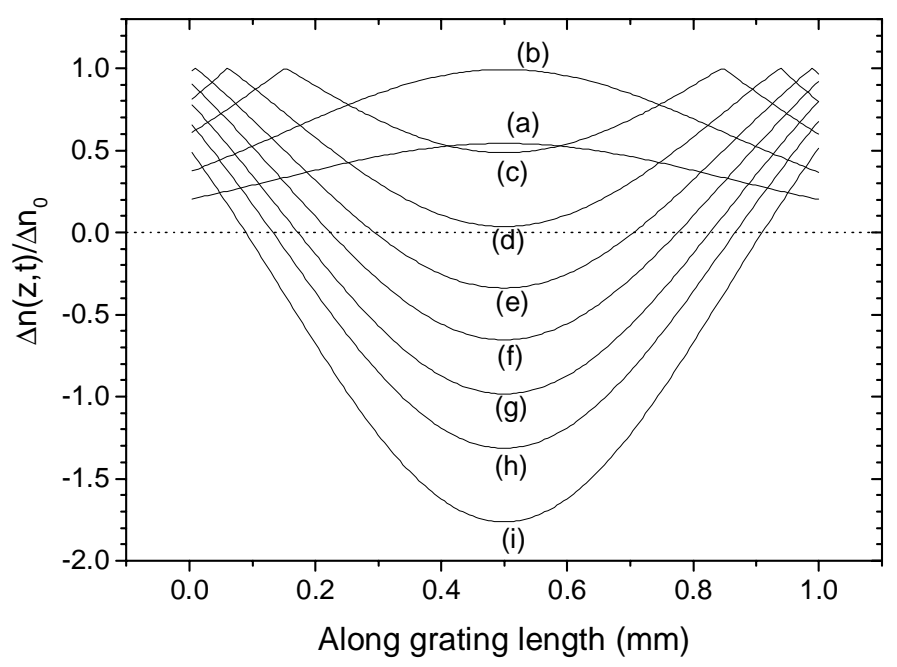

Fig.2 Normalized index change distribution for the simulations used in Fig.3. From (a) to (i), t= 30, 55, 90, 120, 145, $166,188,210,240$ s. 
with positive index change. We can, thus, assume the dynamic UV-induced index distribution across an UV-exposed fibre exhibiting a distribution as

$$
\Delta n(z, t)=\left\{\begin{array}{c}
\Delta n_{0} f(z) \int_{0}^{t} C_{1}(t) d t \quad \text { when } \Delta n(z, t)<\Delta n_{0} \\
\Delta n_{0}\left[1-f(z) \int_{t_{0}(z)}^{t} C_{2}(t) d t\right] \text { when } \Delta n(z, t) \geq \Delta n_{0}
\end{array}\right.
$$

where $f(z)$ is normalized beam profile $\left(f(z)=\exp \left(-B z^{2} / L_{g}^{2}\right)\right.$ with $\mathrm{B}=4$ used in our calculation), $L_{g}$ is the grating length, $z$ is the axis along the grating length, $n_{0}$ is the maximum refractive index change that can be reached for type I grating, $t_{0}$ $(z)$ is the time to reach $n_{0}$ at $z$ position, and $C_{1}(t)$ and $C_{2}(t)$ are the coefficients that indicate the growth speed of type I grating and type IIA grating, respectively. The values of $C_{1}(t)$ and $C_{2}$ $(t)$ are dependent on the UV laser power, and usually are a function of time due to their non-linear growth speed. For simplicity, we have considered $C_{l}(t)$ and $C_{2}(t)$ to be constants (i.e. linear growth regime) in this paper.

Fig. 2 shows a set of normalized index change distributions at different times of $30 \mathrm{~s}, 55 \mathrm{~s}, 90 \mathrm{~s}, 120 \mathrm{~s}, 145 \mathrm{~s}, 166 \mathrm{~s}, 188 \mathrm{~s}$, $210 \mathrm{~s}, 240 \mathrm{~s}$, which were chosen to simulate the growth curves of (a)-(i) in Fig1. In the simulation, other parameters used are $n_{0}=0.001, \quad L g=1 \mathrm{~mm}$, $C_{1}=0.018 \mathrm{~s}^{-1}, C_{2}=0.015 \mathrm{~s}^{-1}$. The calculated transmission spectra with the index change profiles shown in Fig. 2 are plotted in Fig.3. Note, the simulated

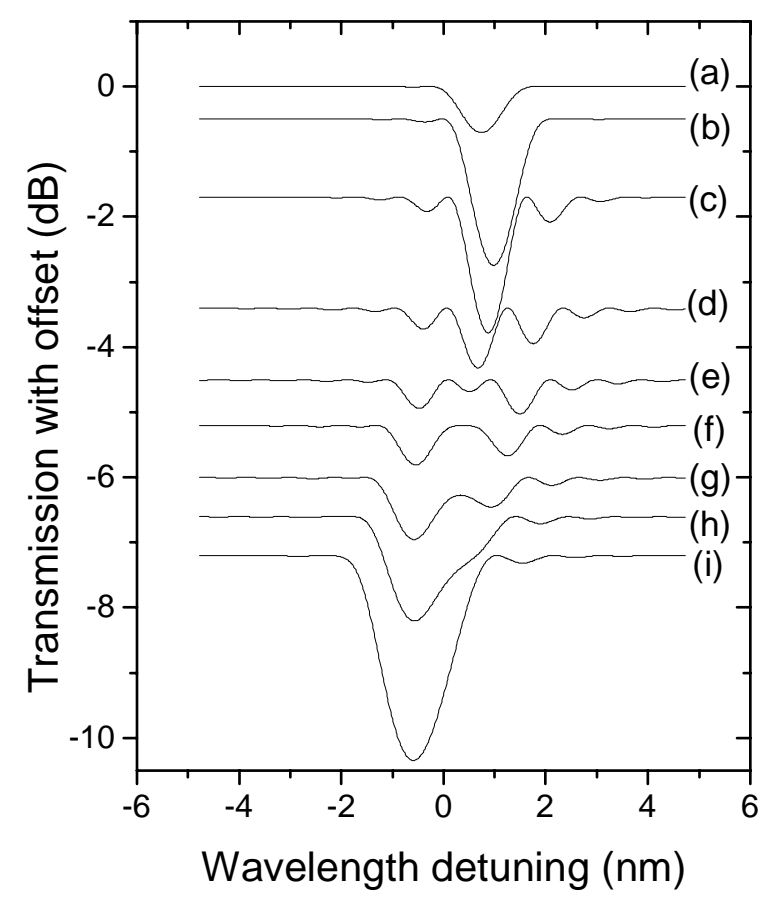

Fig.3 Calculated evolution spectra of a hybrid-type grating during its growth. grating growth curves are in excellent agreement with the experimentally measured ones in Fig. 1, indicating the effectiveness of our model.

Hybrid-type fiber grating may find applications in optical communications and optical sensing. For example, proper combination of positive-index (Type I) and negative-index (Type IIA) grating structures may generate desirable apodization effect leading to either near-constant dispersion [5] or dispersion slop to suit for optical communication applications. Simultaneous temperature and strain sensors may be constructed by mixing different type of gratings, since different type gratings have quite different thermal properties [6].

\section{CONCLUSIONS}

We have introduced a new concept of hybrid-type fiber Bragg grating. As an example, we demonstrate complex growth behaviour of a hybrid-type grating generated in $\mathrm{B} / \mathrm{Ge}$ codoped fiber. A new numerical 
model has been developed to simulate the grating evolution process giving excellent results. This model can be used as a guidance tool for designing gratings with complex index profiles. We anticipate that the hybrid-type gratings may find roles in a number of applications.

\section{ACKNOLEDGEMENTS}

This work was carried out with support of the UK EPSRC research grant.

\section{REFERENCES}

[1] Hill, K.O., and Meltz, G., "Fiber Bragg grating technology fundamentals and overview," J. Lightwave Technol., 1997, 15,(8) pp1263-1276.

[2] Kashyap, R., Fiber Bragg grating. New York: Academic Press, 1999.

[3] Douay M., Xie W.X., Taunay T., Bernage, Niay P., Cordier P., Poumellec B., Dong L., Bayon J.F., Poignant H. and Delevaque E., "Densification involved in the UV-based photosensitivity of silica glasses and optical fibers," J. Lightwave Technol., 1997, 15, pp. 1329-1342.

[4] Xie, W.X., Niay, P., Bernage, P., Douay, M., Bayon, J.F., Georges, T., Monerie, M., and Poumellec, B., "Experimental evidence of two types of photorefractive effects occurring during photoinscriptions of Bragg gratings within germanosilicate fibers," Opt. Comm., 1993, 104, pp.185-195

[5] Ibsen, M., Durkin M.K., Cole M.J., Laming R.I., Electron. Lett., 1998, 34, (8), pp.800-802

[6] Shu, X., Liu, Y., Zhao, D., Gwandu, B., Floreani, F., Zhang, L., and Bennion, I., "Dependence of temperature and strain coefficients on fiber grating type and its application to simultaneous temperature and strain measurement," Opt. Lett., 2002, 27, (9),pp.701-703 